\title{
Conformational Changes of the Small Ribosomal Subunit During Elongation Factor G-dependent tRNA-mRNA Translocation
}

\author{
Frank Peske1, Andreas Savelsbergh', Vladimir I. Katunin ${ }^{2}$ \\ Marina V. Rodnina ${ }^{3}$ and Wolfgang Wintermeyer ${ }^{1 *}$
}

${ }^{1}$ Institute of Molecular Biology University of Witten/Herdecke 58448 Witten, Germany

${ }^{2}$ Petersburg Nuclear Physics Institute, 188355 Gatchina

Russia

${ }^{3}$ Institute of Physical Biochemistry, University of Witten/Herdecke, 58448 Witten Germany

\begin{abstract}
Translocation, a coordinated movement of two tRNAs together with mRNA on the ribosome, is catalyzed by elongation factor G (EF-G). The reaction is accompanied by conformational rearrangements of the ribosome that are, as yet, not well characterized. Here, we analyze those rearrangements by restricting the conformational flexibility of the ribosome by antibiotics binding to specific sites of the ribosome. Paromomycin (Par), viomycin (Vio), spectinomycin (Spc), and hygromycin B (HygB) inhibited the tRNA-mRNA movement, while the other partial reactions of translocation, including the unlocking rearrangement of the ribosome that precedes tRNA-mRNA movement, were not affected. The functional cycle of EF-G, i.e. binding of EF-G.GTP to the ribosome, GTP hydrolysis, Pi release, and dissociation of EF-G.GDP from the ribosome, was not affected either, indicating that EF-G turnover is not coupled directly to tRNAmRNA movement. The inhibition of translocation by Par and Vio is attributed to the stabilization of tRNA binding in the A site, whereas Spc and HygB had a direct inhibitory effect on tRNA-mRNA movement. Streptomycin (Str) had essentially no effect on translocation, although it caused a large increase in tRNA affinity to the A site. These results suggest that conformational changes in the vicinity of the decoding region at the binding sites of Spc and HygB are important for tRNA-mRNA movement, whereas Str seems to stabilize a conformation of the ribosome that is prone to rapid translocation, thereby compensating the effect on tRNA affinity.
\end{abstract}

(C) 2004 Elsevier Ltd. All rights reserved.

Keywords: rapid kinetics; fluorescence; translation; antibiotics; regulation

\section{Introduction}

Translocation is a step in protein synthesis during which two tRNAs bound to the mRNA move a large distance on the ribosome. The reaction is promoted by elongation factor G (EF-G), a GTP-binding protein that hydrolyzes GTP during the reaction. Translocation is much slower without GTP

Abbreviations used: EF-G, elongation factor G; aatRNA, aminoacyl-tRNA; Pmn, puromycin; Str, streptomycin; Tet, tetracycline; Spc, spectinomycin; Par, paromomycin; HygB, hygromycin B; Vio, viomycin; Ery, erythromycin; Lin, linezolid; Sps, sparsomycin; MDCC, 7diethylamino-3-(((2-maleimidyl)ethyl) amino)carbonyl)coumarin; PBP, phosphate-binding protein; cryo-EM, cryo-electron microscopy; Prf, proflavin.

E-mail address of the corresponding author: winterme@uni-wh.de hydrolysis, ${ }^{1,2}$ and extremely slow, but significant, without EF-G under certain conditions in vitro. ${ }^{3,4}$ Prior to translocation, peptidyl-tRNA and deacylated tRNA are bound to the $\mathrm{A}$ and $\mathrm{P}$ sites of the ribosome, respectively. There are extensive contacts between tRNAs and various regions of the ribosome: in the decoding site where residues of $16 \mathrm{~S}$ rRNA interact with the codon-anticodon duplex and the anticodon arms of the tRNAs; ${ }^{5-8}$ at the peptidyl transferase center where residues of $23 \mathrm{~S}$ rRNA form base-specific interactions with the $3^{\prime}$ terminal CCA sequences of both $\mathrm{A}$ and P-site tRNAs; ${ }^{5,9-12}$ and at the subunit interface where bridges that connect the subunits also contact the tRNAs. $5,7,8$ These interactions have to be disrupted, in order to allow movement, and re-established after translocation. This implies that structural elements of the ribosome have to move and rearrange in the course of reaction. 
Detailed structural information as to how and in which sequence structural changes occur during translocation is lacking. Cryo-electron microscopy (cryo-EM) indicates large-scale structural rearrangements of the ribosome that are induced by EF-G. ${ }^{13-16}$ The most extensive changes are seen in the $30 \mathrm{~S}$ subunit, where the arrangement of the head relative to the body changes, ${ }^{14}$ and the relative orientation of the subunits also changes upon EF-G binding, ${ }^{13,15,16}$ suggesting rearrangements at the subunit interface. Chemical footprinting revealed an EF-G-induced conformational change in the head domain involving residues of helix $34^{17}$ in the vicinity of the mRNA in the decoding center. ${ }^{6,18,19}$ Conformational rearrangements of the L7/12 stalk of the $50 \mathrm{~S}$ subunit have been implicated in translocation, ${ }^{20}$ and cryo-EM has revealed different arrangements of the stalk in ribosome-EF-G complexes in different biochemical states. ${ }^{13,14}$

Translocation is a complex process that entails several elemental steps, as identified and characterized by biochemical and rapid kinetic techniques (Figure 1). ${ }^{1,2,21}$ EF-G.GTP binds to the ribosome rapidly and reversibly. Subsequent rapid hydrolysis of GTP causes a coupled conformational change of EF-G and the ribosome (unlocking) that facilitates tRNA-mRNA movement. On the basis of kinetic analyses, movement is rapid intrinsically and instantaneously follows the unlocking rearrangement of the ribosome. The two steps, unlocking and translocation, are physically distinct events, because translocation can be inhibited without affecting the unlocking rearrangement. ${ }^{21,22} \mathrm{Pi}$ is released from EF-G in parallel with and independently of translocation, and the order of the two reactions, translocation and $\mathrm{Pi}$ release, is random. Following Pi release, EF-G changes the conformation from the GDP.Pi to the GDP form (B. Wilden, M.V.R. \& W.W., unpublished results). Further conformational changes of both EF-G and the ribosome take place before EF-G dissociates; some of these changes have been visualized by cryo-EM. ${ }^{14,16,23}$ At the end of translocation, the ribosome is in the post-translocation state with peptidyl-tRNA in the $\mathrm{P}$ site and an overall structure characteristic for the ground state of the ribosome.

Here, we examined which conformational changes of the ribosome are important for translocation. We interfered with the conformational mobility of the ribosome by using antibiotics and studied the effect of antibiotic binding on the elemental steps of translocation. For most of the antibiotics used, crystallographic studies have revealed the molecular details of the interactions with ribosomal residues. ${ }^{24-28}$ Generally, the antibiotics bind to rRNA and stabilize or induce a particular conformation of the region they are bound to. A number of antibiotics are known to inhibit or block translocation by association with specific sites on the ribosome. The antibiotics
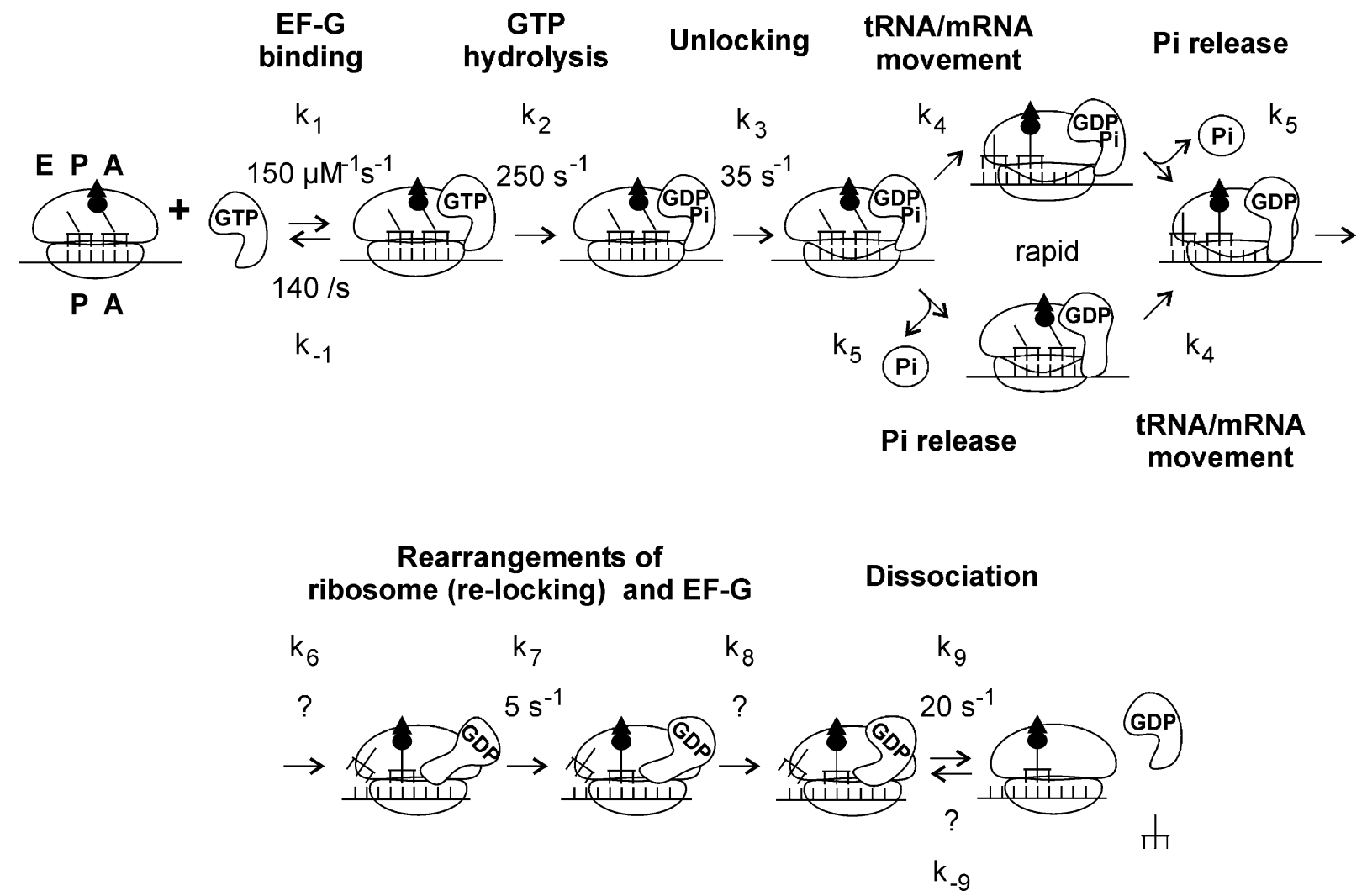

Figure 1. Kinetic model of translocation. Ribosomes are depicted in two conformations, closed and open, to indicate unlocking. EF-G is depicted in different conformations and orientations on the ribosome, based on cryo-EM models. ${ }^{14-16}$ Values of $k_{7}$ and $k_{9}$ are preliminary (B. Wilden, A.S., W.W. \& M.V.R., unpublished results). 
selected for the $30 \mathrm{~S}$ subunit bind to regions of $16 \mathrm{~S}$ rRNA that were shown to be involved, or were implicated, in translocation, i.e. to helix 34 in the 30 $\mathrm{S}$ head (spectinomycin, Spc, and tetracycline, Tet), helix 44 in the decoding site (paromomycin, Par, viomycin, Vio, and hygromycin $\mathrm{B}, \mathrm{HygB}$ ), or in the vicinity of helix 27 (streptomycin, Str, and tetracycline, Tet). A number of antibiotics bind at, or in close proximity to, the peptidyltransferase center on the $50 \mathrm{~S}$ subunit, and many of them inhibit peptide bond formation, either by direct competition within the active site or by interfering with structural changes (erythromycin, Ery; linezolid, Lin; sparsomycin, Sps). Since such changes may affect the movement of the $3^{\prime}$ ends of tRNAs within the active site, those antibiotics were studied also for their effects on translocation.

\section{Results}

\section{Multiple turnover translocation}

The effect of antibiotics on translocation was first examined under conditions of multiple turnover of EF-G. ${ }^{29}$ Pretranslocation complexes with deacylated RNA $^{\text {fMet }}$ in the $P$ site and $\mathrm{f}\left[{ }^{3} \mathrm{H}\right] \mathrm{Met}\left[{ }^{14} \mathrm{C}\right]$ Phe-tRNA ${ }^{\text {Phe }}$ in the A site (Materials and Methods) were mixed with catalytic amounts of EF-G. The displacement of fMetPhe-tRNA ${ }^{\text {Phe }}$ from the $\mathrm{A}$ to the $\mathrm{P}$ site was monitored by the reaction of $\mathrm{P}$ site-bound fMetPhe-tRNA ${ }^{\text {Phe }}$ with puromycin (Pmn) forming the tripeptide fMetPhePmn (Materials and Methods). As expected, ${ }^{30-37}$ translocation was blocked by Par, HygB, and Vio, whereas Spc had a smaller effect (Figure 2(a)). Other antibiotics that have not been reported as translocation inhibitors influenced translocation; however, the effect on both rate (fourfold at most) and extent (twofold) of translocation was moderate (Figure 2(b)). Lin had no significant effect.

Multiple-turnover translocation may be affected by changes at any elemental step of translocation (Figure 1). To understand the mechanism of action of antibiotics, and to correlate antibiotic-induced structural changes with effects on translocation, further experiments were performed under singleround conditions where elemental steps of translocation could be monitored individually.

\section{tRNA-mRNA Movement}

The movement of the tRNA-mRNA complex through the ribosome during translocation was monitored kinetically by fluorescence stopped-flow, using an mRNA labeled with fluorescein at the $3^{\prime}$ end (Figure 3(a)). ${ }^{21}$ Pretranslocation complexes with deacylated tRNA ${ }^{\text {fMet }}$ in the $\mathrm{P}$ site and fMetPhe-tRNA $^{\text {Phe }}$ in the A site were prepared using a fluorescein-labeled mRNA fragment with a coding sequence of 14 nucleotides. Upon mRNA displacement during translocation, the fluorescence increased due to movement of the dye towards the
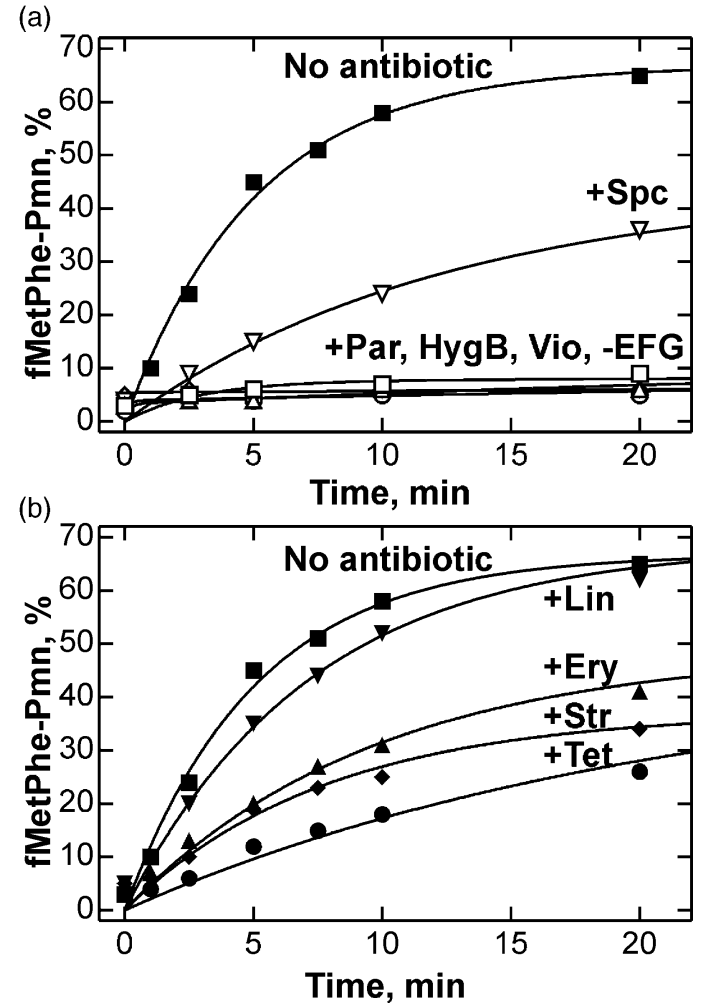

Figure 2. Multiple-turnover translocation. Timecourses of translocation were monitored by reaction with Pmn. Pretranslocation complexes $(0.2 \mu \mathrm{M})$ were stabilized by addition of $\mathrm{Mg}^{2+}$ to $14 \mathrm{mM}$ and translocation was measured after addition of $0.5 \mathrm{nM}$ EF-G. (a) Effect of Spc, Par, HygB, and Vio. (b) Effect of Str, Tet, Ery, and Lin.

mRNA entrance channel of the ribosome. ${ }^{19}$ Upon addition of EF-G at saturating concentrations, ${ }^{21}$ rapid mRNA translocation was observed (Figure 3(b)). Spc, Par, and HygB inhibited the rate of translocation strongly, while the final level of translocation was unchanged (Figure 3(b) and (c); Table 1), and Vio abolished translocation completely (data not shown) ${ }^{1,21}$ Str and Ery decreased the rate of translocation twofold, whereas Tet, Lin, and Sps did not affect the reaction appreciably. The same results were obtained when translocation was monitored by fluorescence changes of proflavin in fMetPhe-tRNA $^{\text {Phe }}(\operatorname{Prf16} / 17)^{1}$ (data not shown).

Detailed analysis of the time-courses showed that the fluorescence change, monitored with either fluorescent mRNA or tRNA, was biphasic, with $>85 \%$ of the amplitude due to a rapid reaction, $16 \mathrm{~s}^{-1}$, and $<15 \%$ to a slower reaction, $0.5 \mathrm{~s}^{-1}$ (Figure 3(d)). Upon addition of Spc in increasing concentration, the amplitude of the slow reaction increased at the cost of the rapid reaction, while the rates of both reactions did not change (Figure 3(e)). At saturating Spc, the fast reaction was virtually absent and $>80 \%$ of the signal change was due to the slow reaction. These data suggest that there are two alternative conformations of the 
(a)
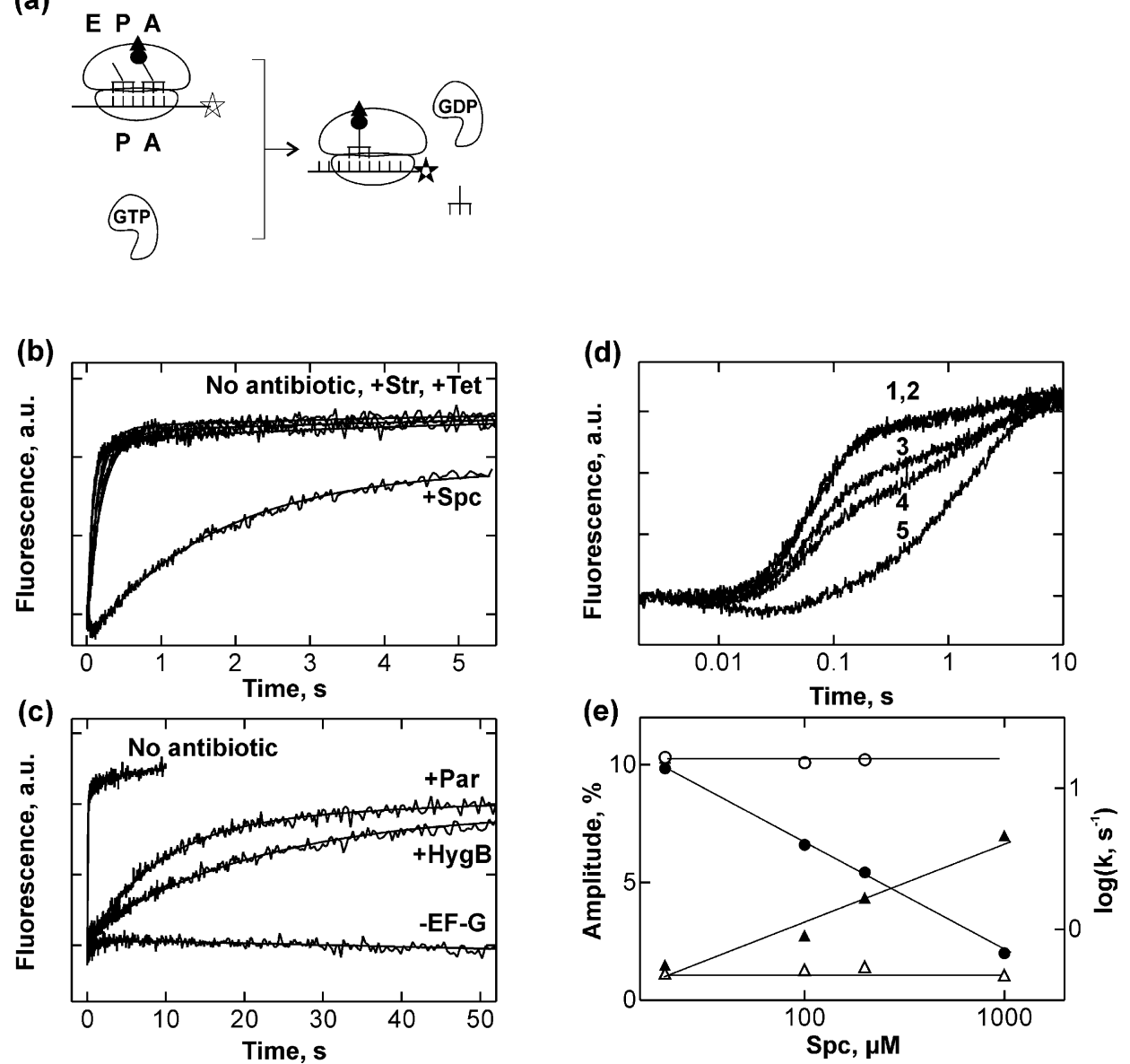

Figure 3. tRNA-mRNA movement. (a) Experimental approach. Pretranslocation complexes containing fluoresceinlabeled mRNA (star) were rapidly mixed in a stopped-flow apparatus with excess saturation concentrations of EF-G to $0.1 \mu \mathrm{M}$ and $2 \mu \mathrm{M}$ final concentrations, respectively. Fluorescence change of fluorescein upon translocation was measured. (b) Time-courses of movement in the absence of antibiotic and in the presence of Str, Tet, or Spc. (c) Timecourses of movement in the presence of Par and HygB. (d) Time-courses of translocation in the absence of antibiotic (1) or in the presence of Spc; $20 \mu \mathrm{M}(2), 100 \mu \mathrm{M}$ (3), $200 \mu \mathrm{M}$ (4), and $1000 \mu \mathrm{M}$ (5) antibiotic. The time-courses are biphasic, with a fast $\left(16 \mathrm{~s}^{-1}\right)$ and a slow $\left(0.5 \mathrm{~s}^{-1}\right)$ reaction. (e) Spc-dependent changes of the amplitudes (filled symbols) and apparent rate constants (open symbols) of fast (circles) and slow (triangles) reactions.

pretranslocation complex that differ in their ability to undergo rapid translocation. In the absence of antibiotic, most of the ribosomes $(>85 \%)$ are capable of rapid translocation and only a small portion $(<15 \%)$ is slow, whereas binding of Spc

Table 1. Effect of antibiotics on the rate of single-round translocation

\begin{tabular}{lcc}
\hline Antibiotic & Rate $\left(\mathrm{s}^{-1}\right)$ & Fold decrease \\
\hline None & 16 & - \\
Str & 7 & 2.3 \\
Tet & 10 & 1.6 \\
Spc & 0.5 & 32 \\
Par & 0.1 & 160 \\
HygB & 0.05 & 320 \\
Vio & $<0.001$ & $>10,000$ \\
Ery & 8 & 2 \\
Lin & 12 & 1.3 \\
Sps & 15 & 1 \\
\hline
\end{tabular}

The rates given are averages of two to four independent experiments. Standard deviations are $<15 \%$. appears to shift the conformational equilibrium towards the slowly translocating form of the ribosomes.

\section{Phosphate release}

Antibiotics may affect translocation by several mechanisms. They may interfere with EF-G binding or with conformational changes required for any of the elemental steps prior to tRNA-mRNA movement depicted in Figure 1, i.e. GTP hydrolysis or ribosome unlocking. To identify the step that is affected, we measured the rate of phosphate (Pi) release from EF-G following GTP hydrolysis and ribosome unlocking (Figure 1), monitoring the fluorescence change of 7-diethylamino-3-(()(2maleimidyl)ethyl) amino)carbonyl)coumarin (MDCC)-labeled phosphate-binding protein (PBP). ${ }^{21}$ None of the antibiotics influenced significantly the rate of $\mathrm{Pi}$ release in the first round (Figure 4(a)) or subsequent multiple rounds (Figure 4(b)). This indicates that the steps up to, 

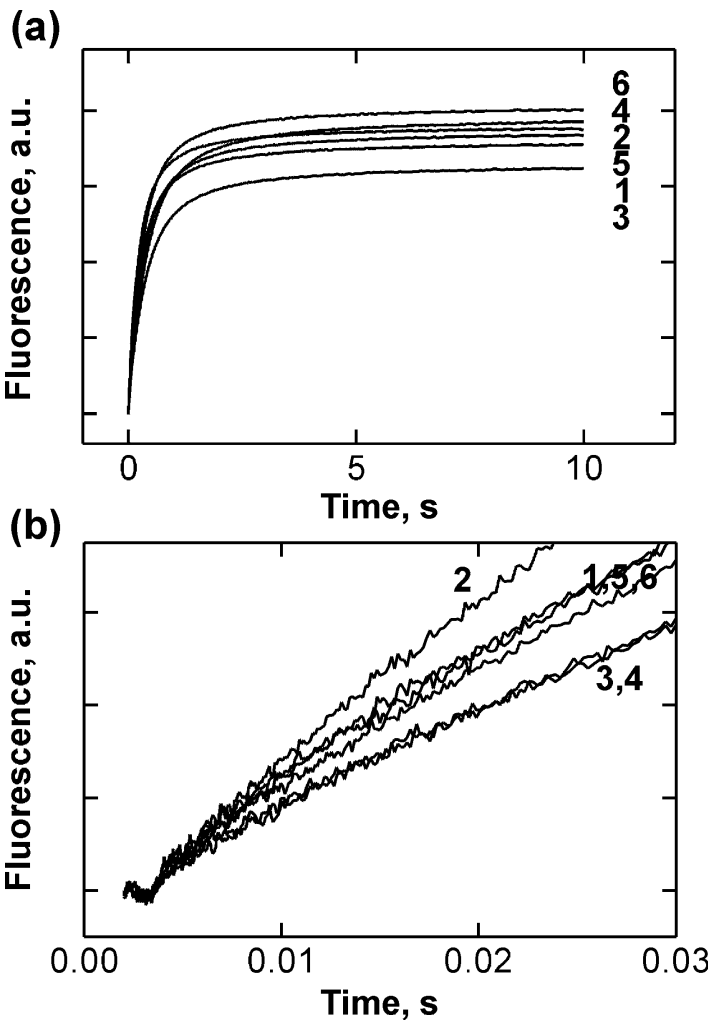

Figure 4. Time-courses of Pi release. (a) Long time window. The rate of multiple turnover was $2.5( \pm 0.5) \mathrm{s}^{-1}$ in all cases. (b) Single-round Pi release; rate constants were $15( \pm 5) \mathrm{s}^{-1}$. Concentrations of the ribosomes and EF-G were $0.5 \mu \mathrm{M}$ and $0.5 \mu \mathrm{M}$, respectively. Pi release in the absence of antibiotics (1), or in the presence of Str (2), Tet (3), Spc (4), Par (5), or HygB (6).

and including, Pi release are not affected by any of the antibiotics studied here, and that tRNA-mRNA movement is the only step that is inhibited.

\section{Stability of peptidyl-tRNA binding in the A site}

The codon-anticodon complex interacts with ribosomal residues in the decoding center, thereby stabilizing tRNA binding in the A site. ${ }^{6}$ Antibiotics that bind to the $30 \mathrm{~S}$ subunit may further stabilize the binding. ${ }^{38-40}$ This would result in the stabilization of the ribosome-tRNA-mRNA complex prior to translocation and lead to a higher-energy barrier of translocation; hence the observed decrease in the rate of translocation. To quantify the effect of antibiotics on the stability of fMetPhe-tRNA ${ }^{\text {Phe }}$ binding, we used the following experimental approach. ${ }^{41,42}$ Pretranslocation complexes were prepared and purified at elevated concentrations of $\mathrm{Mg}^{2+}$ as described in Materials and Methods. Dissociation of $\left.\mathrm{f}^{3} \mathrm{H}\right] \mathrm{Met}\left[{ }^{14} \mathrm{C}\right] \mathrm{Phe}-\mathrm{tRNA}{ }^{\text {Phe }}$ was initiated by diluting the complexes into a buffer with a low concentration of $\mathrm{Mg}^{2+}(7 \mathrm{mM})$. Timecourses of dissociation were followed until the new equilibrium was established (Figure 5). To account for peptidyl-tRNA hydrolysis during prolonged incubation times, fMetPhe formed by hydrolysis was determined by extraction into ethylacetate. Rate constants, $k_{\text {off }}$ and $k_{\text {on }}$ of peptidyl-tRNA interaction with the $\mathrm{A}$ site were calculated by numerical integration from the combined timecourses of dissociation and hydrolysis (Materials and Methods). The equilibrium constant of fMetPhe-tRNA $^{\text {Phe }}$ dissociation from the A site, $K_{d}$, was calculated from the dissociation and association rate constants determined in the absence of antibiotics or in the presence of Spc, Str, and HygB, i.e. the antibiotics that affected the rate of translocation. The affinity of peptidyl-tRNA to the A site in the presence of Par has been determined. ${ }^{41}$

The antibiotics stabilized fMetPhe-tRNA in the A site between 210-fold and 30-fold in the order Par $>$ Str $>$ HygB (Table 2). The stabilization was due to both slower dissociation from the A site and faster rebinding of $\mathrm{tRNA}$ to the ribosome in the presence of antibiotic. In terms of free energy, the ground state of translocation with respect to A-site binding was stabilized by $2.1-3.2 \mathrm{kcal} / \mathrm{mol}$ (Table $3 ; 1 \mathrm{cal}=$ $4.184 \mathrm{~J})$. This effect alone, without any specific effects on ribosome flexibility, may inhibit translocation considerably. Interestingly, Spc weakened the binding of fMetPhe-tRNA ${ }^{\text {Phe }}$ to the A site by $0.8 \mathrm{kcal} / \mathrm{mol}$, thereby slightly destabilizing the ground state of translocation.

\section{Effects of antibiotics on hybrid state formation of peptidyl-tRNA on the $50 \mathrm{~S}$ subunit}

Movement of tRNA during translocation proceeds through a number of intermediate states. ${ }^{43}$ In an early intermediate, the $3^{\prime}$ end of the A site-bound peptidyl-tRNA becomes flexible and samples the $P$ site on the $50 \mathrm{~S}$ subunit (A/P hybrid state) where it can react with Pmn, albeit at a very low rate. ${ }^{4,44,45}$ Antibiotics may impair hybrid state formation by restricting the mobility of the $3^{\prime}$ end. To test this possibility, we measured the reaction of fMetPhetRNA with Pmn in the absence of EF-G (Figure 6). Spc, Vio, and HygB did not affect the slow Pmn reaction, which took place at a rate of $0.002-$ $0.004 \mathrm{~s}^{-1}$ at the concentration of Pmn used (1 mM). Str had a small inhibitory effect. Interestingly, Par reduced the rate of the reaction about fourfold, to $0.0007 \mathrm{~s}^{-1}$, suggesting a long-range conformational effect by the antibiotic which is, however, too small to explain the inhibition of translocation by Par.

\section{Discussion}

\section{Effects of antibiotics on translocation on the 30 $S$ and $50 \mathrm{~S}$ subunits}

There are two possible mechanisms by which antibiotics may inhibit translocation. Antibiotic binding may stabilize tRNA in the respective binding site on the ribosome, i.e. stabilize the pretranslocation state (ground-state stabilization), thereby increasing the energy barrier of 
(a)

(b)

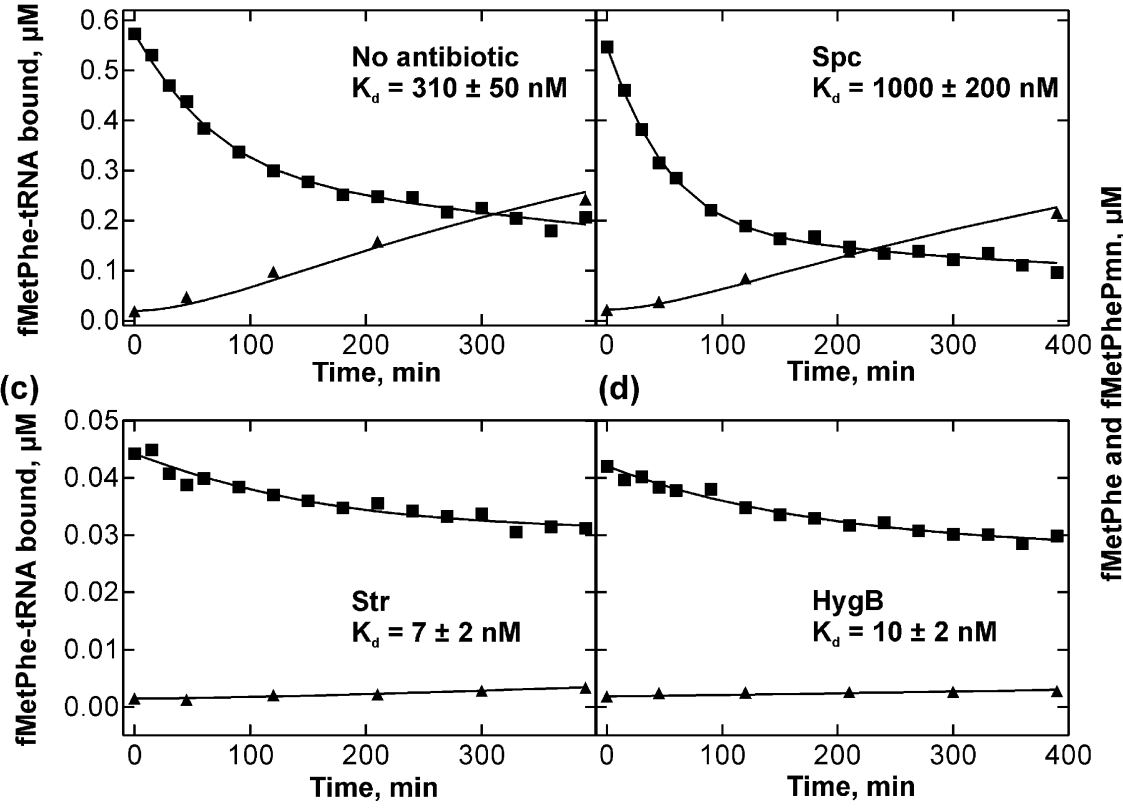

Figure 5. $\mathrm{fMetPhe}_{\mathrm{T} N \mathrm{~A}^{\mathrm{Phe}}}$ dissociation from the A site. Time-courses of fMetPhe-tRNA ${ }^{\text {Phe }}$ dissociation from the ribosome (squares) were measured at conditions of translocation experiments $\left(7 \mathrm{mM} \mathrm{Mg}{ }^{2+}, 37^{\circ} \mathrm{C}\right)$ by nitrocellulose filtration. The amount of fMetPhe-tRNA hydrolyzed during the incubation time and the extent of the spontaneous translocation was measured by extraction of both $\mathrm{fMetPhe}$ and fMetPhe-Pmn into ethylacetate (triangles). (a) In the absence of antibiotics. (b) Spc. (c) Str. (d) HygB.

translocation. Alternatively, antibiotics may block conformational changes of the ribosome that are required for tRNA movement.

Several antibiotics that bind to the $30 \mathrm{~S}$ subunit, including Par, $\mathrm{HygB}, \mathrm{Spc}$, and Vio, inhibited translocation strongly; Vio binds also to the $50 \mathrm{~S}$ subunit. ${ }^{46,47}$ Par, HygB, and Str increase the affinity of tRNA binding in the A site, i.e. stabilize the pretranslocation state of the ribosome. (The faster binding of tRNA to the A site observed in the presence of those antibiotics suggests that the activation energy of tRNA binding is compensated, in part, by antibiotic binding, which may stabilize a particular rRNA conformation that facilitates tRNA binding.) The free energy of stabilization of the pretranslocation state caused by antibiotic binding can be compared to the concomitant increase in the activation energy of translocation (Table 3 ). If the inhibition of translocation were due solely to ground-state stabilization, the decrease of $\Delta \Delta G^{\circ}$ is expected to be equal to the increase of $\Delta \Delta G^{\neq}$, and $\Delta \Delta G^{\circ}+\Delta \Delta G^{\neq}$should be zero. This is in fact the case for Par and Vio, which increased the affinity of tRNA to the A site 210-fold and >1000-fold, ${ }^{48}$ while translocation is inhibited by a factor of 160 and $>10,000$, respectively. HygB stabilized tRNA in the A site, but to an extent that does not fully explain its effect on the translocation rate. Spc destabilized pept-tRNA in the A site, which should decrease the activation energy of translocation and facilitate tRNA movement; however, instead of an increase, a decrease in the translocation rate is observed. This indicates an additional specific effect on translocation that presumably originates from the restricted flexibility of the $30 \mathrm{~S}$ subunit caused by the binding of the antibiotics. Finally, Str, which stabilized tRNA quite considerably, reduced the rate of translocation only marginally (about

Table 2. Effect of antibiotics on A-site binding of peptidyl-tRNA

\begin{tabular}{|c|c|c|c|c|}
\hline \multirow[b]{2}{*}{ Antibiotic } & \multicolumn{2}{|c|}{ Rate constants $^{\mathrm{a}}$} & \multirow[b]{2}{*}{$K_{\mathrm{d}}(\mathrm{nM})$} & \multirow[b]{2}{*}{ Affinity increase } \\
\hline & $\begin{array}{l}\text { Association } \\
\left(\mu \mathrm{M}^{-1} \mathrm{~h}^{-1}\right)\end{array}$ & Dissociation $\left(\mathrm{h}^{-1}\right)$ & & \\
\hline None & 1.64 & 0.57 & 310 & - \\
\hline Str & 15.2 & 0.10 & 7 & 45 \\
\hline Spc & 0.63 & 0.68 & 1000 & 0.3 \\
\hline HygB & 10.3 & 0.11 & 10 & 30 \\
\hline Par & $200^{b}$ & $0.30^{\mathrm{b}}$ & $1.5^{\mathrm{c}}$ & 210 \\
\hline
\end{tabular}


Table 3. Free energy $(\mathrm{kcal} / \mathrm{mol})$ of A-site binding of peptidyl-tRNA $\left(\Delta G^{\circ}\right)$ and free energy of activation of translocation $\left(\Delta G^{\neq}\right)$

\begin{tabular}{|c|c|c|c|c|c|}
\hline Antibiotic & $\Delta G^{\circ}$ & $\Delta G^{\neq}$ & $\Delta \Delta G^{\circ \mathrm{a}}$ & $\Delta \Delta G^{\neq}$ & $\Delta \Delta G^{\circ}+\Delta \Delta G^{\neq}$ \\
\hline None & -5.0 & 16.5 & - & - & \\
\hline Str & -7.3 & 16.9 & -2.3 & 0.4 & -1.9 \\
\hline Spc & -4.2 & 18.6 & +0.8 & 2.1 & 2.9 \\
\hline HygB & -7.1 & 20.0 & -2.1 & 3.5 & 1.4 \\
\hline Par & -8.2 & 19.6 & -3.2 & 3.1 & -0.1 \\
\hline
\end{tabular}

a $\Delta \Delta G^{\circ}$ and $\Delta \Delta G^{\neq}$represent the differences of the respective values of $\Delta G^{\circ}$ and $\Delta G^{\neq}$determined in the presence and in the absence of antibiotic. Standard deviations were below $20 \%$, except $\Delta G^{\circ}$ with Par, which was $25 \%$.

twofold). This may be explained by assuming that Str induces a conformation of the $30 \mathrm{~S}$ subunit that is more prone to translocation, thereby compensating the unfavorable effect of ground-state stabilization by lowering the activation energy barrier. In conclusion, $\mathrm{HygB}, \mathrm{Spc}$, and Str seem to influence translocation by affecting the conformational dynamics of the $30 \mathrm{~S}$ subunit.

As a result of peptide bond formation on the $50 \mathrm{~S}$ subunit, the $3^{\prime}$ end of the A-site tRNA seems to gain some flexibility and can move over to the $\mathrm{P}$ site, leading to the formation of the A/P hybrid state, as indicated by footprinting and biochemical studies. ${ }^{4,42,43,45}$ This movement on the $50 \mathrm{~S}$ subunit appears to be largely independent of the movement of the anticodon domain of the tRNA on the $30 \mathrm{~S}$ subunit, as antibiotics that inhibited translocation strongly by binding to the $30 \mathrm{~S}$ subunit had no or little effect on hybrid state formation. Aa-tRNA, which does not adopt the hybrid state, is translocated 130 times more slowly than peptidyltRNA. ${ }^{42}$ Conformational changes related to the formation of the hybrid state are likely to be subtle and reversible, and could not be visualized by lowresolution cryo-EM. ${ }^{16}$ Binding of Sps to the A site on the $50 \mathrm{~S}$ subunit stimulated spontaneous translocation in the absence of EF-G, possibly by restricting the backward movement of the $3^{\prime}$ end of the tRNA. ${ }^{37}$ However, in the presence of EF-G, stimulation of translocation by the antibiotic is

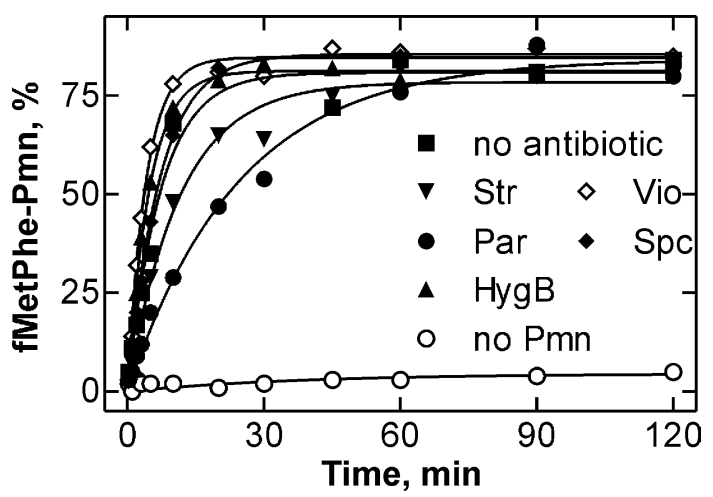

Figure 6. Pmn reactivity of peptidyl-tRNA in the A/P hybrid state. Pretranslocation complexes $(0.2 \mu \mathrm{M}, 100 \%)$ in the absence or in the presence of antibiotics were incubated with Pmn $(1 \mathrm{mM})$ in the absence of EF-G. No spontaneous translocation was observed within the incubation time (up to 120 minutes; data not shown). ${ }^{4,44,45}$ insignificant. Furthermore, cryo-EM analysis showed that the $3^{\prime}$ end of the P-site tRNA changed its position upon EF-G binding and moved into the E site, thereby adopting a stable hybrid state. ${ }^{16}$ This suggest that the factor may act similarly to Sps in promoting translocation on the $50 \mathrm{~S}$ subunit, either by stabilizing the $3^{\prime}$ ends of the two tRNAs in the post-translocation position or by favoring the unidirectionality of movement.

\section{Conformational changes of the $30 \mathrm{~S}$ subunit in translocation}

In the following, structural changes of the $30 \mathrm{~S}$ subunit required for translocation are discussed on the basis of the crystal structures of 30 S-antibiotic complexes. $^{24,25}$

\section{Hygromycin B}

HygB has a single binding site on the $30 \mathrm{~S}$ subunit that is located at the very top of helix 44 . The antibiotic makes contacts to nucleotides in both strands of 16 S rRNA in the region 1490-1500 and $1400-1410 .^{24}$ Resistance to HygB is caused by impaired binding of HygB to the $30 \mathrm{~S}$ subunit that is caused by mutations at the HygB binding site, ${ }^{49}$ or the disruption of the G1491:C1409 base-pair outside the binding site. ${ }^{50}$ Binding of HygB does not seem to induce any significant alterations in the structure of $16 \mathrm{~S}$ rRNA. ${ }^{24}$ The antibiotic interacts with bases of $16 \mathrm{~S}$ rRNA that contact the tRNAs in both $\mathrm{A}$ and $\mathrm{P}$ sites. The part of helix 44 to which HygB binds has been implicated in movements during translocation. ${ }^{51,52}$ There are several ways in which the mobility of this region of helix 44 may be involved in $\mathrm{tRNA}$ displacement. The tRNA-mRNA complex has extensive contacts to $16 \mathrm{~S}$ rRNA in the A site decoding center. ${ }^{6}$ Movement of $16 \mathrm{~S}$ rRNA in the complex with tRNA-mRNA into the direction of the P site may occur, at least partly, without the disruption of these contacts. ${ }^{52}$ According to that model, EF-G induces a conformation of the $16 \mathrm{~S}$ rRNA that participates actively in tRNA movement. Alternatively, a conformational change of helix 44, in particular in the region where HygB binds, may be necessary to disrupt the interactions between the codon-anticodon complex and $16 \mathrm{~S}$ rRNA prior to movement and/or to vacate the space between the $\mathrm{A}$ and $\mathrm{P}$ sites to allow for tRNA-mRNA movement 
independently of $16 \mathrm{~S}$ rRNA. A direct steric hindrance for tRNA displacement by HygB, without invoking conformational changes of helix 44 , is less likely, because HygB is bound in the major groove of helix 44 , on the side opposite the codonanticodon complex. An additional stabilization of the $\mathrm{P}$ site-bound tRNA through the contacts between HygB and U1498 in 16 S rRNA, $^{24}$ and potentially the mRNA in the $\mathrm{P}$ site, leading to inhibition of the tRNA movement from the P to the E site, cannot be excluded.

\section{Spectinomycin}

Spc binds in the minor groove at one end of helix 34, where it contacts $\mathrm{C} 1064$ and $\mathrm{C} 1192 .{ }^{25}$ Mutations of these $16 \mathrm{~S}$ rRNA residues impair Spc binding and lead to Spc resistance. ${ }^{53-55}$ However, mutations in the ribosomal protein S5, which does not make direct contacts with Spc, were also shown to cause resistance to Spc. ${ }^{55,56}$ These mutations map to a loop of S5 that stabilizes interactions within the central pseudoknot, helix 28, 35, and $36 .{ }^{25}$ Although the crystal structure shows no contact of Spc with that region, it is possible that such interactions take place in other $30 \mathrm{~S}$ conformations. The present data (Figure 3(d) and (e)) suggest that Spc stabilizes a conformation of the $70 \mathrm{~S}$ pretranslocation complex that is present, albeit at low frequency, also in the absence of the antibiotic and is intrinsically slow in translocation. Mutations in S5 that cause resistance to Spc may shift the equilibrium between the two structures towards the rapidly translocating one even in the presence of Spc. Translocation is likely to involve movement of elements of the head of the $30 \mathrm{~S}$ subunit, as indicated by cryo-EM reconstructions. $^{13,14,57}$ The movement of the head may be involved in tRNA displacement directly or indirectly, by affecting the structure or orientation of structural elements of the $30 \mathrm{~S}$ subunit that interact with the $50 \mathrm{~S}$ subunit (intersubunit bridges). ${ }^{5,58}$ Interestingly, another antibiotic that binds to helix 34, Tet, did not inhibit translocation at all. The Tet binding site is located between helices 34 and 31 of $16 \mathrm{~S}$ rRNA, ${ }^{24}$ further away from helices 28,35 , and 36 , as compared to Spc. UV cross-linking suggested that both antibiotics induce subtle longrange effects on the $30 \mathrm{~S}$ subunits. ${ }^{59}$ However, the $30 \mathrm{~S}$ structures stabilized by Tet and Spc are probably different, as they have different functional effects on translation.

\section{Streptomycin}

The antibiotic binds between helices 1, 18, 27, 28, and 44 of $16 \mathrm{~S}$ rRNA protein $S 12 .{ }^{25}$ It connects and immobilizes the shoulder (S12 and G530 loop) and the central part of the subunit (helices 27/44). By increasing the number of interactions between these regions, streptomycin moves the head towards the shoulder of the $30 \mathrm{~S}$ subunit, but into a position different from that induced by cognate codonanticodon recognition. ${ }^{60}$ The antibiotic stabilizes
aminoacyl-tRNA binding in the A site and alters the rates of GTP hydrolysis by elongation factor $\mathrm{Tu}$ in a reciprocal way on cognate and near-cognate codons, resulting in almost identical rates of GTP hydrolysis for cognate and near-cognate ternary complexes. ${ }^{39}$ Str decreases the rate of translocation only twofold, despite the 45-fold tRNA stabilization in the A site. This suggests that Str freezes the $30 \mathrm{~S}$ subunit in a conformation that is inherently more prone to rapid translocation, and this effect may compensate the increased activation energy of translocation that results from ground-state stabilization. Thus, the conformation that the $30 \mathrm{~S}$ subunit assumes upon binding of $\operatorname{Str}^{25}$ may show structural features that are characteristic for the transition state of translocation during which tRNA movement takes place.

\section{Ribosome unlocking and tRNA translocation}

The cryo-EM reconstructions show that EF-G binding to ribosomes or ribosome-tRNA complexes induces conformational changes of the ribosome, ${ }^{14,16,57}$ which involve a rearrangement between the subunits, changes in the positions of the L1 and L7/12 stalks, and changes of the $30 \mathrm{~S}$ subunit. It was reported that the binding of EFG.GDPNP required the presence of deacylated tRNA in the P site and does not take place when there is peptidyl-tRNA in the $\mathrm{P}$ site. ${ }^{61}$ From this observation, it was deduced that the ability of P sitebound tRNA to adopt the $\mathrm{P} / \mathrm{E}$ hybrid state is a requirement for the binding of EF-G in the GTP form (mimicked by GDPNP), and called the state after peptidyl transfer the unlocked state of the ribosome, although cryo-EM did not reveal a conformational change of the ribosome. ${ }^{16,61}$ However, when the interaction of EF-G.GTP with ribosomes was studied kinetically, the rate of EF-G binding and both the rate and extent of GTP hydrolysis were the same regardless of whether charged or uncharged tRNA was bound to the P site or whether the A site was vacant or occupied. ${ }^{1}$ These results indicated that the presence of charged tRNA in the $\mathrm{P}$ site $(\mathrm{P} / \mathrm{P}$ state) does not interfere with ribosome binding of EF-G.GTP and GTP hydrolysis, whereas the rate of translocation is decreased. ${ }^{62}$ We attribute the different observations to differences between GTP and GDPNP, in keeping with the 50-fold inhibition of EF-G-dependent translocation that is observed when GTP is replaced with GDPNP, other non-hydrolyzable GTP analogs, or GDP. ${ }^{1,2}$ According to the kinetic analysis, ${ }^{21}$ the slow translocation is due to the inhibition of a conformational rearrangement of the ribosome that is induced by EF-G binding and strongly accelerated by GTP hydrolysis. That rearrangement was referred to as unlocking, ${ }^{21}$ in order to indicate that it constitutes a global structural change of the ribosome that presumably includes changes at the subunit interface such as those observed by cryoEM. ${ }^{16,23}$ Aside from the different use of the term unlocking, the principal difference between the two 
models is whether unlocking of the ribosome and tRNA-mRNA movement constitute a single rearrangement, ${ }^{16}$ or whether unlocking and movement are two physically distinct steps, as indicated by rapid kinetic and biochemical analysis (Figure 1). ${ }^{21}$

The present data show that several antibiotics inhibited the movement of $t$ RNAs on the ribosome, whereas other steps of translocation, including the release of phosphate, were not affected. This indicates strongly that none of the antibiotics tested interferes with the unlocking rearrangement of the ribosome that rate-limits both tRNA movement and Pi release, and supports the view that ribosome unlocking and tRNA movement are two physically distinct steps. ${ }^{21}$ In the absence of antibiotics, the rate of unlocking limits the following steps of translocation and Pi release; therefore, the steps are not separated kinetically. Furthermore, the present data indicate that the functional cycle of EF-G (binding, GTP hydrolysis, Pi release, and dissociation) is independent on tRNA movement. This suggests that movement can be inhibited without interfering with unlocking, indicating that movement is a step after unlocking.

These results have important implications for understanding the mechanism by which EF-G catalyzes tRNA translocation. If EF-G were a deterministic motor that moves actively the tRNAs through the ribosome, perhaps coupled to rearrangements of the ribosome, one would expect GTP hydrolysis to be coupled to the movement via release of Pi. Such a model of EF-G function is disfavoured for the following reasons. First, as described above, the conformational rearrangements of EF-G and the ribosome that are induced by GTP hydrolysis are independent of tRNA movement, indicating the lack of direct coupling. Second, rapid translocation requires GTP hydrolysis, but not Pi release, indicating indirect coupling between chemical and mechanical steps. ${ }^{21}$ Finally, translocation can occur spontaneously without EFG, albeit very slowly, or with EF-G but without GTP hydrolysis, i.e. under conditions where movement is due to thermal motion only. ${ }^{1,2,58}$ These facts are reconciled more easily in a scenario in which EF-G utilizes the energy of GTP hydrolysis to induce a rearrangement of the ribosome and subsequently, presumably driven by $\mathrm{Pi}$ release, biases forward movement, which itself takes place by spontaneous diffusion enabled by the unlocking rearrangement. Thus, we favor a model in which EF-G has two functions in translocation catalysis. One, carried out by EF-G.GDP.Pi, is to impose conformational strain upon the ribosome in order to promote unlocking, which is the prerequisite for tRNA movement. The second is to bias diffusion to produce forward movement. The first function is driven by GTP hydrolysis directly, i.e. can be referred to as mechanochemical function, while the other involves a reorientation of EF-G.GDP that presumably is induced by a conformational change accompanying Pi release, resembling the function of a Brownian ratchet.

\section{Materials and Methods}

\section{Materials}

Experiments were carried out in buffer A ( $50 \mathrm{mM}$ Tris$\mathrm{HCl}(\mathrm{pH} 7.5), 70 \mathrm{mM} \mathrm{NH}_{4} \mathrm{Cl}, 30 \mathrm{mM} \mathrm{KCl}, 7 \mathrm{mM} \mathrm{MgCl}_{2}$ ) at $37^{\circ} \mathrm{C}$. To prepare fluorescently labeled mRNA (54 nt, coding sequence of $14 \mathrm{nt}$ starting with fMetPhe...), MFmRNA was oxidized by potassium periodate and reacted with fluorescein-5-thiosemicarbazide (Molecular Probes) ${ }^{21} \mathrm{f}\left[{ }^{3} \mathrm{H}\right]$ Met-tRNA ${ }^{\mathrm{fMet}}$ (Escherichia coli), $\left[{ }^{14} \mathrm{C}\right] \mathrm{Phe}-$ tRNA $^{\text {Phe }}$ (yeast), and tRNA ${ }^{\text {Phe }}(\operatorname{Prf16} / 17)$ (yeast) were prepared as described, ${ }^{63,64}$ as were ribosomes from $E$. coli MRE600, MF-mRNA, initiation factors, EF-Tu, and EFG. ${ }^{65}$ MDCC-labeled PBP was prepared as described. ${ }^{21}$ Antibiotics were from Sigma or Fluka and were used at the following concentrations: Par, $5 \mu \mathrm{M}$; HygB, $20 \mu \mathrm{M}$; Vio, $200 \mu \mathrm{M}$; Spc, $1 \mathrm{mM}$; Str, $20 \mu \mathrm{M}$; Tet, $50 \mu \mathrm{M}$; Sps, $250 \mu \mathrm{M}$; Ery, $200 \mu \mathrm{M}$; Lin, $250 \mu \mathrm{M}$ (a kind gift from Bayer HealthCare AG).

\section{Biochemical assays}

The preparation of pretranslocation complexes programmed with MF-mRNA (or fluorescein-labeled MFmRNA) and carrying deacylated tRNA ${ }^{\text {fMet }}$ in the P site and fMetPhe-tRNA ${ }^{\text {Phe }}$ in the A site has been described. ${ }^{21}$ Ribosomes $(1 \mu \mathrm{M})$ were incubated with a twofold excess of MF-mRNA in the presence of $1.5 \mu \mathrm{M}$ initiation factors IF1, IF2, IF3, $1.5 \mu \mathrm{M}$ f $\left[{ }^{3} \mathrm{H}\right]$ Met-tRNA ${ }^{\text {fMet }}$, and $1 \mathrm{mM}$ GTP in buffer $\mathrm{A}\left(7 \mathrm{mM} \mathrm{Mg}{ }^{2+}\right)$ for one hour at $37^{\circ} \mathrm{C}$. Ternary complex, EF-Tu.GTP. $\left[{ }^{14} \mathrm{C}\right]$ Phe-tRNA ${ }^{\text {Phe }}$, was prepared by incubating $10 \mu \mathrm{M}$ EF-Tu with $1 \mathrm{mM}$ GTP, $3 \mathrm{mM}$ phosphoenol pyruvate, $0.1 \mathrm{mg} / 1$ of pyruvate kinase for 15 minutes at $37^{\circ} \mathrm{C}$, followed by addition of $5 \mu \mathrm{M}$ $\left[{ }^{14} \mathrm{C}\right]$ Phe-tRNA ${ }^{\text {Phe }}$. Ternary complex was added to the initiation complex and incubated for $30 \mathrm{~s}$ at $20^{\circ} \mathrm{C}$ to form the pretranslocation complex carrying fMetPhe-tRNA ${ }^{\text {Phe }}$ in the $\mathrm{A}$ site. Then, the concentration of $\mathrm{Mg}^{2+}$ was adjusted to $20 \mathrm{mM}$ to prevent drop-off of fMetPhetRNA $^{\text {Phe }}$ from the A site. Pretranslocation complexes were purified by ultracentrifugation through $400 \mu \mathrm{l}$ of a 1.1 M sucrose cushion in buffer A with $20 \mathrm{mM} \mathrm{Mg}^{2+}$ for two hours at $259,000 \mathrm{~g}$ in a Sorvall M120GX centrifuge. The amount of $\left[{ }^{14} \mathrm{C}\right]$ Phe and $\left[{ }^{3} \mathrm{H}\right]$ Met bound to ribosomes was determined by nitrocellulose filtration by applying aliquots of the reaction mixture to the filters $(0.45 \mu \mathrm{m}$, Sartorius) and subsequent washing with $5 \mathrm{ml}$ of buffer A. Filters were dissolved and radioactivity measured in QS361 scintillation cocktail (Zinsser Analytic): $>95 \%$ of the ribosomes carried the tRNAs as indicated. To induce translocation, EF-G, preincubated with $1 \mathrm{mM}$ of GTP for 15 minutes at $37^{\circ} \mathrm{C}$, was mixed with pretranslocation complex at the indicated concentrations. The extent of translocation $(>85 \%)$ was determined by reaction with Pmn (1 mM Pmn, $\left.10 \mathrm{~s}, 37^{\circ} \mathrm{C}\right)$. $^{1}$ Hybrid state formation of $\mathrm{fMetPhe} \mathrm{tRNA}{ }^{\text {Phe }}$ on the $50 \mathrm{~S}$ subunit was measured in the absence of EF-G upon prolonged incubations of pretranslocation complexes with $1 \mathrm{mM} \mathrm{Pmn}$ at $37^{\circ} \mathrm{C}$ in buffer A with $14 \mathrm{mM} \mathrm{MgCl}_{2}$. Pmn reactions were quenched with $500 \mu \mathrm{l}$ of $1.5 \mathrm{M}$ sodium acetate saturated with $\mathrm{MgSO}_{4}$. After addition of $750 \mu \mathrm{l}$ of ethyl acetate, extraction for five minutes at room temperature, and phase separation by centrifugation, $500 \mu \mathrm{l}$ of the organic 
phase was taken for counting in Luma Safe Plus (LumacLSC). Rates of Pi release were measured in the presence of $200 \mu \mathrm{M}$ GTP and $2.5 \mu \mathrm{M}$ MDCC-labeled PBP. $^{21}$

To initiate the dissociation of fMetPhe-tRNA ${ }^{\text {Phe }}$ from the A site, the concentration of $\mathrm{Mg}^{2+}$ was adjusted as indicated, and pept-tRNA bound to the A site after incubation for different times was determined by nitrocellulose filtration. Dissociation time-courses were evaluated by numerical integration, using the Scientist software (Micromath Scientific Software) as described. ${ }^{41}$

\section{Rapid kinetics}

Fluorescence stopped-flow measurements were performed and the data evaluated as described. ${ }^{1,21}$ Fluorescein and proflavin were excited at $470 \mathrm{~nm}$, and the fluorescence was measured after passing a KV500 cut-off filter (Schott); MDCC fluorescence was excited at $425 \mathrm{~nm}$ and measured after passing a KV450 filter. Experiments were performed in buffer $\mathrm{A}$ at $37^{\circ} \mathrm{C}$ by rapidly mixing equal volumes $(50 \mu \mathrm{l})$ each of the pretranslocation complex $(0.2 \mu \mathrm{M}$ in the syringe $)$ and EF-G.GTP $(4 \mu \mathrm{M}$ in the syringe). The data were evaluated by exponential fitting using TableCurve software (Jandel Scientific).

\section{Acknowledgements}

We thank Carmen Schillings, Astrid Böhm, Simone Möbitz and Petra Striebeck for expert technical assistance, and Kerstin Ehlert, Bayer HealthCare, for a kind gift of linezolid. The work was supported by the Deutsche Forschungsgemeinschaft, the European Union, the Alfried Krupp von Bohlen und Halbach-Stiftung, the Russian Foundation for Basic Research, the International Bureau of the Bundesministerium für Bildung und Forschung, and the Fonds der Chemischen Industrie.

\section{References}

1. Rodnina, M. V., Savelsbergh, A., Katunin, V. I. \& Wintermeyer, W. (1997). Hydrolysis of GTP by elongation factor $G$ drives tRNA movement on the ribosome. Nature, 385, 37-41.

2. Katunin, V. I., Savelsbergh, A., Rodnina, M. V. \& Wintermeyer, W. (2002). Coupling of GTP hydrolysis by elongation factor $G$ to translocation and factor recycling on the ribosome. Biochemistry, 41, 12806-12812.

3. Gavrilova, L. P., Kostiashkina, O. E., Koteliansky, V. E., Rutkevitch, N. M. \& Spirin, A. S. (1976). Factor-free ("non-enzymic") and factor-dependent systems of translation of polyuridylic acid by Escherichia coli ribosomes. J. Mol. Biol. 101, 537-552.

4. Sharma, D., Southworth, D. R. \& Green, R. (2004). EFG-independent reactivity of a pre-translocation-state ribosome complex with the aminoacyl tRNA substrate puromycin supports an intermediate (hybrid) state of tRNA binding. RNA, 10, 102-113.

5. Yusupov, M. M., Yusupova, G. Z., Baucom, A.,
Lieberman, K., Earnest, T. N., Cate, J. H. \& Noller, H. F. (2001). Crystal structure of the ribosome at $5.5 \AA$ resolution. Science, 292, 883-896.

6. Ogle, J. M., Brodersen, D. E., Clemons, W. M., Tarry, M. J., Carter, A. P. \& Ramakrishnan, V. (2001). Recognition of cognate transfer RNA by the $30 \mathrm{~S}$ ribosomal subunit. Science, 292, 897-902.

7. Valle, M., Sengupta, J., Swami, N. K., Grassucci, R. A., Burkhardt, N., Nierhaus, K. H. et al. (2002). Cryo-EM reveals an active role for aminoacyl-tRNA in the accommodation process. EMBO J. 21, 3557-3567.

8. Stark, H., Rodnina, M. V., Wieden, H.-J., Zemlin, F., Wintermeyer, W. \& van Heel, M. (2002). Ribosome interactions of aminoacyl-tRNA and elongation factor $\mathrm{Tu}$ in the codon recognition complex. Nature Struct. Biol. 9, 849-854.

9. Samaha, R. R., Green, R. \& Noller, H. F. (1995). A base pair between tRNA and 23S rRNA in the peptidyl transferase centre of the ribosome. Nature, 377, 309-314.

10. Kim, D. F. \& Green, R. (1999). Base-pairing between 23S rRNA and tRNA in the ribosomal A site. Mol. Cell, 4, 859-864.

11. Nissen, P., Hansen, J., Ban, N., Moore, P. B. \& Steitz, T. A. (2000). The structural basis of ribosome activity in peptide bond synthesis. Science, 289, 920-930.

12. Schmeing, T. M., Seila, A. C., Hansen, J. L., Freeborn, B., Soukup, J. K., Scaringe, S. A. et al. (2002). A pretranslocational intermediate in protein synthesis observed in crystals of enzymatically active $50 \mathrm{~S}$ subunits. Nature Struct. Biol. 9, 225-230.

13. Frank, J. \& Agrawal, R. K. (2000). A ratchet-like intersubunit reorganization of the ribosome during translocation. Nature, 406, 318-322.

14. Stark, H., Rodnina, M. V., Wieden, H.-J., van Heel, M. \& Wintermeyer, W. (2000). Large-scale movement of elongation factor $\mathrm{G}$ and extensive conformational change of the ribosome during translocation. Cell, 100, 301-309.

15. Tama, F., Valle, M., Frank, J. \& Brooks, C. L. (2003). Dynamic reorganization of the functionally active ribosome explored by normal mode analysis and cryo-electron microscopy. Proc. Natl Acad. Sci. USA, 100, 9319-9323.

16. Valle, M., Zavialov, A., Sengupta, J., Rawat, U., Ehrenberg, M. \& Frank, J. (2003). Locking and unlocking of ribosomal motions. Cell, 114, 123-134.

17. Matassova, N. B., Rodnina, M. V. \& Wintermeyer, W. (2001). Elongation factor G-induced structural change in helix 34 of $16 \mathrm{~S}$ rRNA related to translocation on the ribosome. RNA, 7, 1879-1885.

18. Sergiev, P. V., Lavrik, I. N., Wlasoff, V. A., Dokudovskaya, S. S., Dontsova, O. A., Bogdanov, A. A. \& Brimacombe, R. (1997). The path of mRNA through the bacterial ribosome: a site-directed crosslinking study using new photoreactive derivatives of guanosine and uridine. RNA, 3, 464-475.

19. Yusupova, G. Z., Yusupov, M. M., Cate, J. H. \& Noller, H. F. (2001). The path of messenger RNA through the ribosome. Cell, 106, 233-241.

20. Traut, R. R., Dey, D., Bochkariov, D. E., Oleinikov, A. V., Jokhadze, G. G., Hamman, B. \& Jameson, D. (1995). Location and domain structure of Escherichia coli ribosomal protein L7/L12: site specific cysteine crosslinking and attachment of fluorescent probes. Biochem. Cell Biol. 73, 949-958.

21. Savelsbergh, A., Katunin, V. I., Mohr, D., Peske, F., Rodnina, M. V. \& Wintermeyer, W. (2003). An 
elongation factor G-induced ribosome rearrangement precedes tRNA-mRNA translocation. Mol. Cell, 11, 1517-1523.

22. Peske, F., Matassova, N. B., Savelsbergh, A., Rodnina, M. V. \& Wintermeyer, W. (2000). Conformationally restricted elongation factor $\mathrm{G}$ retains GTPase activity but is inactive in translocation on the ribosome. Mol. Cell, 6, 501-505.

23. Agrawal, R. K., Penczek, P., Grassucci, R. A. \& Frank, J. (1998). Visualization of elongation factor $G$ on the Escherichia coli $70 S$ ribosome: The mechanism of translocation. Proc. Natl Acad. Sci. USA, 95, 6134-6138.

24. Brodersen, D. E., Clemons, W. M., Carter, A. P., Morgan-Warren, R. J., Wimberly, B. T. \& Ramakrishnan, V. (2000). The structural basis for the action of the antibiotics tetracycline, pactamycin, and hygromycin B on the 30 S ribosomal subunit. Cell, 103, 1143-1154.

25. Carter, A. P., Clemons, W. M., Brodersen, D. E., Morgan-Warren, R. J., Wimberly, B. T. \& Ramakrishnan, V. (2000). Functional insights from the structure of the $30 \mathrm{~S}$ ribosomal subunit and its interactions with antibiotics. Nature, 407, 340-348.

26. Hansen, J. L., Schmeing, T. M., Moore, P. B. \& Steitz, T. A. (2002). Structural insights into peptide bond formation. Proc. Natl Acad. Sci. USA, 99, 11670-11675.

27. Pioletti, M., Schlunzen, F., Harms, J., Zarivach, R., Gluhmann, M., Avila, H. et al. (2001). Crystal structures of complexes of the small ribosomal subunit with tetracycline, edeine and IF3. EMBO J. 20, 1829-1839.

28. Schluenzen, F., Tocilj, A., Zarivach, R., Harms, J., Gluehmann, M., Janell, D. et al. (2000). Structure of functionally activated small ribosomal subunit at 3.3 angstroms resolution. Cell, 102, 615-623.

29. Savelsbergh, A., Matassova, N. B., Rodnina, M. V. \& Wintermeyer, W. (2000). Role of domains 4 and 5 in elongation factor $\mathrm{G}$ functions on the ribosome. J. Mol. Biol. 300, 951-961.

30. Modolell, J. \& Vazquez, D. (1977). The inhibition of ribosomal translocation by viomycin. Eur. J. Biochem. 81, 491-497.

31. Cabanas, M. J., Vazquez, D. \& Modolell, J. (1978). Dual interference of hygromycin B with ribosomal translocation and with aminoacyl-tRNA recognition. Eur. J. Biochem. 87, 21-27.

32. Zierhut, G., Piepersberg, W. \& Böck, A. (1979). Comparative analysis of the effect of aminoglycosides on bacterial protein synthesis in vitro. Eur. J. Biochem. 98, 577-583.

33. Davies, J. \& Davis, B. D. (1968). Misreading of ribonucleic acid code words induced by aminoglycoside antibiotics. The effect of drug concentration. J. Biol. Chem. 243, 3312-3316.

34. Weisblum, B. \& Davies, J. (1968). Antibiotic inhibitors of the bacterial ribosome. Bacteriol. Rev. 32, 493-528.

35. Lodmell, J. S., Gutell, R. R. \& Dahlberg, A. E. (1995). Genetic and comparative analyses reveal an alternative secondary structure in the region of nt 912 of Escherichia coli 16S rRNA. Proc. Natl Acad. Sci. USA, 92, 10555-10559.

36. Bilgin, N., Richter, A. A., Ehrenberg, M., Dahlberg, A. E. \& Kurland, C. G. (1990). Ribosomal RNA and protein mutants resistant to spectinomycin. EMBO J. 9, 735-739.

37. Fredrick, K. \& Noller, H. F. (2003). Catalysis of ribosomal translocation by sparsomycin. Science, 300, 1159-1162.

38. Karimi, R. \& Ehrenberg, M. (1994). Dissociation rate of cognate peptidyl-tRNA from the A-site of hyperaccurate and error-prone ribosomes. Eur. J. Biochem. 226, 355-360.

39. Gromadski, K. \& Rodnina, M. V. (2004). Streptomycin interferes with conformational coupling between codon recognition and GTPase activation on the ribosome. Nature Struct. Mol. Biol. 11, 316-322.

40. Pape, T., Wintermeyer, W. \& Rodnina, M. V. (2000). Conformational switch in the decoding region of $16 \mathrm{~S}$ rRNA during aminoacyl-tRNA selection on the ribosome. Nature Struct. Biol. 7, 104-107.

41. Konevega, A. L., Soboleva, N. G., Makhno, V. I., Semenkov, Y. P., Wintermeyer, W., Rodnina, M. V. \& Katunin, V. I. (2004). Purine bases at position 37 of tRNA stabilize codon-anticodon interaction in the ribosomal A site by stacking and $\mathrm{Mg}^{2+}$-dependent interactions. $R N A, \mathbf{1 0}, 90-101$.

42. Semenkov, Y. P., Rodnina, M. V. \& Wintermeyer, W. (2000). Energetic contribution of tRNA hybrid state formation to translocation catalysis on the ribosome. Nature Struct. Biol. 7, 1027-1031.

43. Moazed, D. \& Noller, H. F. (1989). Intermediate states in the movement of transfer RNA in the ribosome. Nature, 342, 142-148.

44. Semenkov, Y. P., Shapkina, T. G. \& Kirillov, S. V. (1992). Puromycin reaction of the A-site bound peptidyltRNA. Biochimie, 74, 411-417.

45. Semenkov, Y., Shapkina, T., Makhno, V. \& Kirillov, S. (1992). Puromycin reaction for the A site-bound peptidyl-tRNA. FEBS Letters, 296, 207-210.

46. Moazed, D. \& Noller, H. F. (1987). Chloramphenicol, erythromycin, carbomycin and vernamycin B protect overlapping sites in the peptidyl transferase region of 23 S ribosomal RNA. Biochimie, 69, 879-884.

47. Moazed, D. \& Noller, H. F. (1990). Binding of tRNA to the ribosomal $\mathrm{A}$ and $\mathrm{P}$ sites protects two distinct sets of nucleotides in $16 \mathrm{~S}$ rRNA. J. Mol. Biol. 211, 135-145.

48. Wintermeyer, W., Savelsbergh, A., Semenkov, Y. P., Katunin, V. \& Rodnina, M. V. (2001). Mechanism of elongation factor $\mathrm{G}$ function in tRNA translocation on the ribosome. Cold Spring Harbor Symp. Quant. Biol. 66, 449-458.

49. Pfister, P., Risch, M., Brodersen, D. E. \& Böttger, E. C. (2003). Role of 16S rRNA helix 44 in ribosomal resistance to hygromycin B. Antimicrob. Agents Chemother. 47, 1496-1502.

50. De Stasio, E. A. \& Dahlberg, A. E. (1990). Effects of mutagenesis of a conserved base-paired site near the decoding region of Escherichia coli $16 \mathrm{~S}$ ribosomal RNA. J. Mol. Biol. 212, 127-133.

51. Agrawal, R. K., Spahn, C. M., Penczek, P., Grassucci, R. A., Nierhaus, K. H. \& Frank, J. (2000). Visualization of tRNA movements on the Escherichia coli $70 S$ ribosome during the elongation cycle. J. Cell Biol. 150, 447-460.

52. van Loock, M. S., Agrawal, R. K., Gabashvili, I. S., Qi, L., Frank, J. \& Harvey, S. C. (2000). Movement of the decoding degion of the $16 \mathrm{~S}$ ribosomal RNA accompanies tRNA translocation. J. Mol. Biol. 304, 507-515.

53. Sigmund, C. D., Ettayebi, M. \& Morgan, E. A. (1984). Antibiotic resistance mutations in $16 \mathrm{~S}$ and $23 S$ ribosomal RNA genes of Escherichia coli. Nucl. Acids Res. 12, 4653-4663.

54. Brink, M. F., Brink, G., Verbeet, M. P. \& de Boer, H. A. (1994). Spectinomycin interacts specifically with the residues G1064 and C1192 in 16S rRNA, thereby potentially freezing this molecule into an inactive conformation. Nucl. Acids Res. 22, 325-331.

55. Bilgin, N., Richter, A. A., Ehrenberg, M., Dahlberg, 
A. E. \& Kurland, C. G. (1990). Ribosomal RNA and protein mutants resistant to spectinomycin. EMBO J. 9, 735-739.

56. Bollen, A., Davies, J., Ozaki, M. \& Mizushima, S. (1968). Ribosomal protein conferring sensitivity to the antibiotic spectinomycin in Escherichia coli. Science, 165, 85-86.

57. Agrawal, R. K., Heagle, A. B., Penczek, P., Grassucci, R. A. \& Frank, J. (1999). EF-G-dependent GTP hydrolysis induces translocation accompanied by large conformational changes in the 705 ribosome. Nature Struct. Biol. 6, 643-647.

58. Noller, H. F., Yusupov, M. M., Yusupova, G. Z., Baucom, A. \& Cate, J. H. (2002). Translocation of tRNA during protein synthesis. FEBS Letters, 514, $11-16$.

59. Noah, J. W., Dolan, M. A., Babin, P. \& Wollenzien, P. (1999). Effects of tetracycline and spectinomycin on the tertiary structure of ribosomal RNA in the Escherichia coli $30 \mathrm{~S}$ ribosomal subunit. J. Biol. Chem. 274, 16576-16581.

60. Ogle, J. M., Carter, A. P. \& Ramakrishnan, V. (2003).
Insights into the decoding mechanism from recent ribosome structures. Trends Biochem. Sci. 28, 259-266.

61. Zavialov, A. V. \& Ehrenberg, M. (2003). PeptidyltRNA regulates the GTPase activity of translation factors. Cell, 114, 113-122.

62. Lill, R., Robertson, J. M. \& Wintermeyer, W. (1989). Binding of the $3^{\prime}$ terminus of tRNA to $23 \mathrm{~S}$ rRNA in the ribosomal exit site actively promotes translocation. EMBO J. 8, 3933-3938.

63. Rodnina, M. V., Semenkov, Y. P. \& Wintermeyer, W. (1994). Purification of fMet-tRNA(fMet) by fast protein liquid chromatography. Anal. Biochem. 219, 380-381.

64. Rodnina, M. V. \& Wintermeyer, W. (1995). GTP consumption of elongation factor Tu during translation of heteropolymeric mRNAs. Proc. Natl Acad. Sci. USA, 92, 1945-1949.

65. Rodnina, M. V., Savelsbergh, A., Matassova, N. B., Katunin, V. I., Semenkov, Y. P. \& Wintermeyer, W. (1999). Thiostrepton inhibits turnover but not GTP hydrolysis by elongation factor $\mathrm{G}$ on the ribosome. Proc. Natl Acad. Sci. USA, 96, 9586-9590.

Edited by J. Doudna

(Received 25 July 2004; received in revised form 29 August 2004; accepted 30 August 2004) 